\section{Development and Characterization of 15 Microsatellite Loci for Rhododendron delavayi Franch. (Ericaceae)}

\author{
Nian Wang \\ Kunming Institute of Botany, Chinese Academy of Sciences, Kunming \\ 650204, Yunnan, China; and Graduate School of the Chinese Academy of \\ Sciences, Beijing 100049, China
}

Zhang Chang Qin' and Jun-bo Yang
Kunming Botanical Garden, Lanhei Road 132, Kunming Institute of Botany,
Chinese Academy of Sciences, 65 China, Kunming, Yunnan, China

Jing-li Zhang

Yunnan Agricultural University, Kunming 650201, Yunnan, China

\section{Additional index words. Rhododendron delavayi, microsatellite, SSR markers}

\begin{abstract}
Rhododendron delavayi Franch. is an important ornamental plant and often plays a role in natural hybridization with other sympatric species in Rhododendron subgenus Hymenanthes. Fifteen microsatellite loci were developed and characterized in this species. The average allele number of these microsatellites was four per locus, ranging from three to six. The ranges of expected $\left(H_{E}\right)$ and observed $\left(H_{O}\right)$ heterozygosities were 0.0365 to 0.7091 and 0.0263 to 0.9512 , respectively. Cross-species amplification in $\boldsymbol{R}$. agastum and $\boldsymbol{R}$. decorum showed that a subset of these markers holds promise for congeneric species study. These sets of markers are potentially useful to investigate the genetic structure and gene flow of $R$. delavayi and other congeneric species.
\end{abstract}

Rhododendron, renowned for its ornamental value, is one of the largest and most widespread woody plant genera with over 1000 species distributed from the northern temperate zone, throughout tropical southeast Asia to northeastern Australia (Chamberlain, 2003; Cox, 1994; Ng and Corlett, 2000). There are nine subgenera in Rhododendron (Fang et al., 2005), and many species have been introduced and cultivated for ornamental and landscape purposes. Over 1000 rhododendron cultivars have been bred or selected in the world, especially for species from Rhododendron subgenus Hymenanthes (Chamberlain, 1982). Rhododendron delavayi Franch. is an important ornamental tree species with red flowers; it belongs to Rhododendron subgenus Hymenanthes. It distributes from southwest China to southeast Asia, especially in the Himalayan region, and it is often involved in natural hybridization with other sympatric Rhododendron species

\footnotetext{
Received for publication 19 Nov. 2009. Accepted for publication 4 Jan. 2010.

This study was supported by the Nature Science Foundation of China (Grant No. 30770139) and The Ministry of Science and Technology of China (Grant No. 2003BA901A14). This study was conducted at the Key Laboratory of Plant Biodiversity and Biogeography, Kunming Institute of Botany, Chinese Academy of Sciences.

We thank Dr Frédéric M.B. Jacques and Tonjock Rosemary for valuable suggestions on the manuscript and for help in editing English.

${ }^{1}$ To whom reprint requests should be addressed; e-mail zhangchangqin@mail.kib.ac.cn.
}

We sampled $34 R$. delavayi individuals among five populations (two populations were from Yangbi and the remaining three from Kunming, Zhanyi, and Shizong), four $R$. agastum and $R$. decorum individuals among three populations, respectively (the three populations of $R$. agastum and $R$. decorum were from Zhanyi, Shizong, and Yangbi), across Yunnan province, southwest China. Genomic DNA of $R$. delavayi was used for the construction of all genomic libraries. In total, 42 Rhododendron individuals were used to detect SSR loci polymorphism and to test the transferability of microsatellite markers. A microsatellite enriched library was conducted by using a modified biotin-streptavidin capture method (Chen et al., 2008). Briefly, the genomic DNA $(\approx 500$ to $800 \mathrm{ng})$ was completely digested with $M s e I$ restriction enzyme (NEB) and then the digested fragments were ligated to a MseI amplified fragment length polymorphism adaptor followed by amplification with adaptor-specific primers (5' GAT GAG TCC TGA GTA AN-3') (Huang et al., 2009). For enrichment of the fragments $(\approx 300$ to $800 \mathrm{bp}$ ) containing SSR, the polymerase chain reaction (PCR) products were hybridized to a mixture of biotinylated probes $\left[(\mathrm{AAG})_{10},(\mathrm{AC})_{15},(\mathrm{AG})_{15}\right] \quad$ (Zane et al., 2002). The purified PCR products were ligated into PGEM-T vector (Promega, Beijing, China) and transformed into Escherichia coli strain DH5a (Tiangen, Beijing, China). The positive clones were picked out and tested using $(\mathrm{AAG})_{7} /(\mathrm{AC})_{10} /(\mathrm{AG})_{10}$ and SP6/T7 vector primers, respectively. A total of 462 clones were chosen for sequencing with an $\mathrm{ABI}$ PRISM 3730XL SEQUENCER (Shanghai, China). In all, 240 clones (52\%) were found to contain microsatellite sequences. Finally, 90 pairs of SSR primers were selected for primer designing using Primer Premier 5.0 software (Premier, Canada) (Clarke and Gorley, 2001). Primer pairs were assessed in 34 wild $R$. delavayi individual samples from southeast to southwest Yunnan, China. Microsatellite loci were amplified in a final volume of a 15$\mu \mathrm{L}$ reaction containing $7.5 \mu \mathrm{L} 29$ Taq PCR MasterMix [Tiangen; $0.1 \mathrm{U}$ Taq polymerase/ $\mu \mathrm{L}, 0.5 \mathrm{~mm}$ dNTP each, $20 \mathrm{~mm}$ Tris- $\mathrm{HCl}$ (pH 8.3), $100 \mathrm{~mm} \mathrm{KCl,} 3 \mathrm{~mm} \mathrm{MgCl}_{2}$ ], 0.6 $\mu \mathrm{M}$ of each primer, and $\approx 50 \mathrm{ng}$ genomic DNA. The amplification profiles included initial denaturation at $94{ }^{\circ} \mathrm{C}$ for $3 \mathrm{~min}$ followed by 35 to 40 cycles of $30 \mathrm{~s}$ at $94^{\circ} \mathrm{C}, 30 \mathrm{~s}$ at 54 to $68^{\circ} \mathrm{C}$, and $1 \mathrm{~min}$ at $72{ }^{\circ} \mathrm{C}$ and then final extension 
at $72{ }^{\circ} \mathrm{C}$ for $7 \mathrm{~min}$. The amplified products were then separated on $6 \%$ denaturing polyacrylamide gels and visualized by silver staining.
A 20-bp DNA ladder standard (Fermentas, Shenzhen, China) was used as the standard for scoring.
Table 1. Cross-species amplification of two congeneric species, $R$. decorum and $R$. agastum. weak amplification (W), monomorphic amplification (M), polymorphic amplification (P).

\begin{tabular}{|c|c|c|c|}
\hline Locus & Primer sequence $\left(5^{\prime}-3^{\prime}\right)$ & R. decorum & R. agastum \\
\hline \multirow[t]{2}{*}{$\overline{\mathrm{R}}-111$} & F 5' AGCTTGTAGCTTGGTGAG 3' & $\mathrm{M}$ & $\mathrm{P}$ \\
\hline & R 5' TTGACAAGACAATGGGAG 3' & & \\
\hline \multirow[t]{2}{*}{ R-112 } & F 5' TACTGTTCCTTTCCTTCC 3' & $\mathrm{P}$ & $\mathrm{P}$ \\
\hline & R 5' CTCCCACAATGTCTTCTC 3' & & \\
\hline \multirow[t]{2}{*}{$\mathrm{R}-140$} & F 5' GAAGCCAGTGCTGTGATT 3' & $\mathrm{W}$ & M \\
\hline & R 5' CAGCCAACTTTGACCTAA 3' & & \\
\hline \multirow[t]{2}{*}{$\mathrm{R}-147$} & F 5' TAATCATTACTCTTCCTCCTAG 3' & W & $\mathrm{P}$ \\
\hline & R 5' TCCTTTCTCGATCCCTCT 3' & & \\
\hline \multirow[t]{2}{*}{ R-163 } & F 5' CGTGAGAAATCGTCCAAA 3' & $\mathrm{P}$ & $\mathrm{P}$ \\
\hline & R 5' TCCAAAAGGGGAGTCATT 3' & & \\
\hline \multirow[t]{2}{*}{$\mathrm{R}-166$} & F 5' AACGAAACCCAAGAAACT 3' & $\mathrm{P}$ & $\mathrm{P}$ \\
\hline & R 5' TCATTTATCTCCACTCCC 3' & & \\
\hline \multirow[t]{2}{*}{ R-172 } & F 5' GCTGTCGTATTTACCCTAA 3' & $\mathrm{P}$ & M \\
\hline & R 5' AGATGAAGAAGTGCTGGA 3 & & \\
\hline \multirow{2}{*}{ R-210 } & F 5' TTTCTGGCTCAAAGAGGG 3' & M & $\mathrm{P}$ \\
\hline & R 5' ATTTGGCAGTGTTTCTCA 3' & & \\
\hline \multirow[t]{2}{*}{$\mathrm{R}-299$} & F 5' TACTGTGCTTAGACGCCATT 3' & $\mathrm{P}$ & $\mathrm{P}$ \\
\hline & R 5' CCCTCTGCTGTAAAACTG 3' & & \\
\hline \multirow[t]{2}{*}{$\mathrm{R}-318$} & F 5' AGTCTTATCTTCGCTTCC 3' & $\mathrm{M}$ & $\mathrm{P}$ \\
\hline & R 5' ACTATCAACTCGCAGGTC 3' & & \\
\hline \multirow[t]{2}{*}{$\mathrm{R}-320$} & F 5' GCTGATACGGAGCTGGTG 3' & W & M \\
\hline & R 5' GACGCAAATGGGTGAACT 3' & & \\
\hline \multirow[t]{2}{*}{$\mathrm{R}-335$} & F 5' TCAGGCAACCGTAACAAC 3' & W & $\mathrm{P}$ \\
\hline & R 5' AAACGCAACAAACAGCAA 3' & & \\
\hline \multirow[t]{2}{*}{ R-432 } & F 5' CCGTTTGAGTATCTTCCC 3' & $\mathrm{P}$ & $\mathrm{P}$ \\
\hline & R 5' CTGGTCCATTCTCCAAGTA 3' & & \\
\hline \multirow[t]{2}{*}{$\mathrm{R}-544$} & F 5' TCTGGACTTCAAGCAACA 3' & $\mathrm{P}$ & M \\
\hline & R 5' AATGGAGTAAATGGGGTG 3' & & \\
\hline \multirow[t]{2}{*}{$\mathrm{R}-557$} & F 5' CGAAACTCAGAACCTCCG 3 & $\mathrm{P}$ & $\mathrm{P}$ \\
\hline & R 5' TTCCGAACTCCTTCACCAG 3' & & \\
\hline
\end{tabular}

Cross-species amplification of $R$. agastum and $R$. decorum was further investigated using four wild individuals from three populations for each species (Table 1; Fig. 1). From the 22 tested markers, 15 pairs showed clear polymorphic PCR products across five populations of $R$. delavayi and six pairs in $R$. decorum and nine pairs in $R$. agastum, respectively (Table 1 ). The variability at each locus was measured in terms of number of alleles (A), observed $\left(H_{O}\right)$, and expected heterozygosity $\left(H_{E}\right)$ for the 15 microsatellite loci using GENEPOP Version 4.0 (Raymond and Rousset, 1995). The same software was used to test the deviations from HardyWeinberg equilibrium (HWE) and pairwise linkage disequilibrium. The number of alleles per locus (A) was three to six with an average of four; values for observed $\left(H_{O}\right)$ and expected $\left(H_{E}\right)$ heterozygosities ranged from 0.0263 to 0.9512 (averaged at 0.3122 ) and from 0.0365 to 0.7091 (averaged at 0.4032 ), respectively (Table 2). Seven loci (R-111, R-112，R-147，R-299，R-320，R-335, and R-544) deviated significantly from the HWE $(P<0.01)$. No significant linkage disequilibrium was detected between locus pairs except for three locus pairs: R-299 and R-544, R-166 and R-320, and R-111 and R-320. Altogether, these primers can provide a useful tool to investigate genetic relationships between these closely related Rhododendron species. They are also of great potential to study genetic structure, hybridization and evolution of Rhododendron species in this genus.

Table 2. Characteristics of 15 microsatellite loci developed for Rhododendron delavayi.

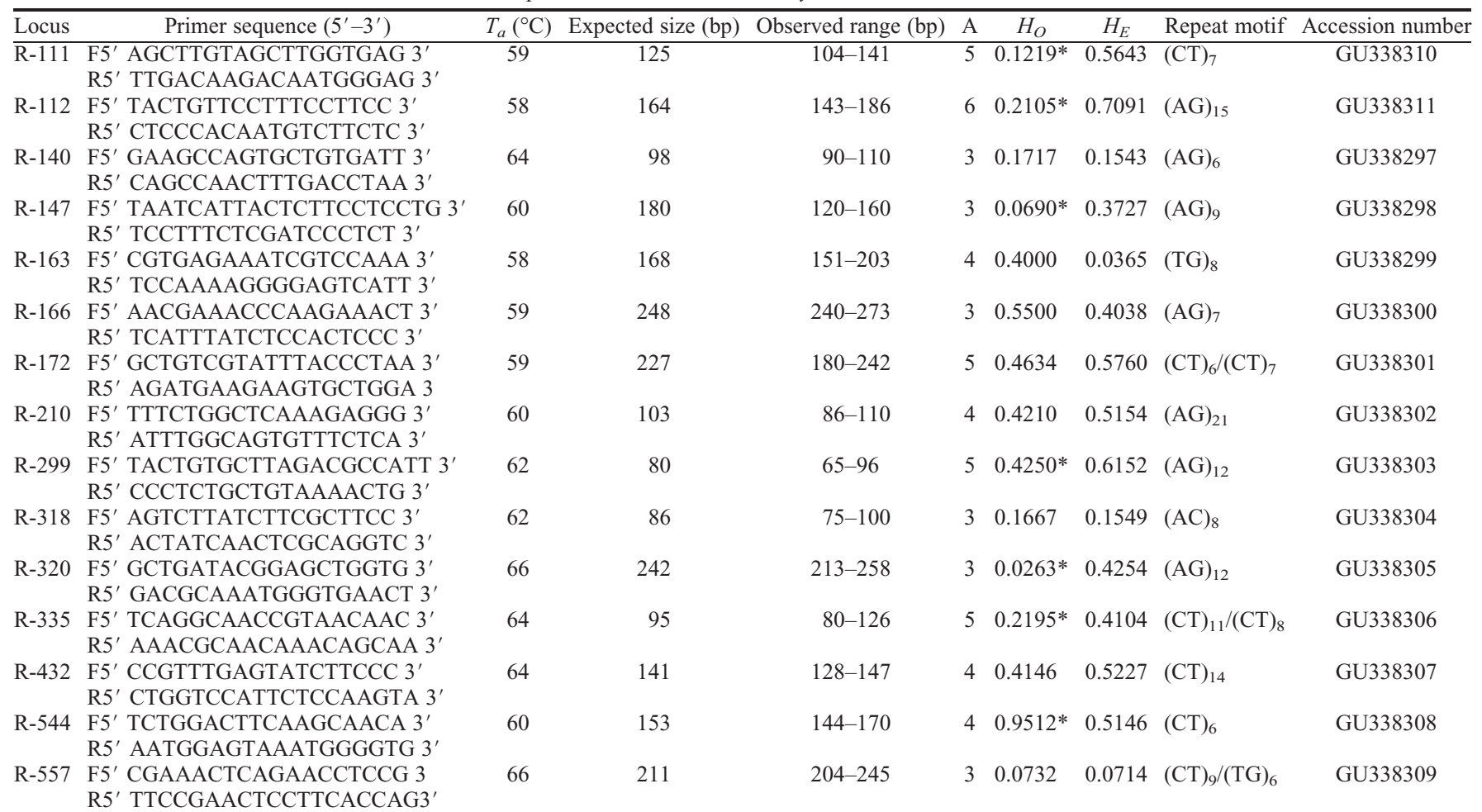

Primer sequence, polymerase chain reaction (PCR) annealing temperature $(\mathrm{Ta})$, expected and observed size range of PCR products, number of observed alleles (A), expected heterozygosity $\left(H_{E}\right)$, and observed heterozygosity $\left(H_{O}\right)$.

"The observed heterozygosity is significantly different from the expected heterozygosity under Hardy-Weinberg equilibrium $(P<0.01)$. 


\section{Literature Cited}

Chamberlain, D.F. 1982. A revision of Rhododendron II. Subgenus Hymenanthes. Notes from the Royal Botanic Garden Edinburgh 39:209-486.

Chamberlain, D.F. 2003. Rhododendrons in the wild: A taxonomist's view, p. 42-52. In: Argent, G. and M. McFarlane (eds.). Rhododendrons in horticulture and science. The Royal Botanic Garden Press, Edinburgh, UK.

Chen, T., R.C. Zhou, X.J. Ge, and S.H. Shi. 2008. Development and characterization of microsatellite markers for a mangrove tree species Sonneratia caseolaris (L.) Egler (Lythraceae sensu lato). Conserv. Genet. 9:957-959.

Clarke, K.R. and R.N. Gorley. 2001. PRIMER v5: User manual/tutorial. PRIMER-E Ltd., Plymouth, UK, p. 91.

Cox, P.A. 1994. Note of natural hybrids and intraspecific variation of Rhododendron in China, p. 132-133. In: Duan, C.Z., S.C. Liao, Q.L. Li, and X.W. Li (eds.). Scientific investigation of the plants on Cangshan Mountain.
Yunnan Science and Technology Press, Kunming, China.

Dikshit, H.K., T. Jhang, N.K. Singh, K.R. Koundal, K.C. Bansal, N. Chandra, J.L. Tickoo, and T.R. Sharma. 2007. Genetic differentiation of Vigna species by RAPD, URP and SSR markers. Biol. Plant. 51:451-457.

Fang, M.Y., R.Z. Fang, M.Y. He, L.Z. Hu, H.B. Yang, H.N. Qin, T.L. Min, F.D. Chamberlain, P. Stevens, G.D. Wallace, and A. Anderberg. 2005. Rhododendron (Ericaceae), p. 260-455. In: Wu, Z.Y. and P.H. Raven (eds.). Flora of China. Vol. 14. Science Press, Beijing, China; Missouri Botanical Garden Press, St. Louis, MO.

Huang, Y., Y. Li, X. Hu, X.J. Ge, C.Q. Zhang, and C.L. Long. 2009. Development of twelve polymorphic microsatellite loci in polyploid endangered Omphalogramma vincaeflora Franch. (Primulaceae). Conserv. Genet. 10:515-517.

Milne, R.I., R.J. Abbott, K. Wolff, and D.F. Chamberlain. 1999. Hybridization among sympatric species of Rhododendron (Ericaceae) in
Turkey: Morphological and molecular evidence. Amer. J. Bot. 86:1776-1785.

Ng, S.C. and R.T. Corlett. 2000. Genetic variation and structure in six Rhododendron species (Ericaceae) with contrasting local distribution patterns in Hong-Kong, China. Mol. Ecol. 9:959-969.

Raymond, M. and F. Rousset. 1995. GENEPOP, v. 1.2: Population genetics software for exact tests and ecumenicism. J. Hered. 86:248-249.

Zane, L., L. Bargellont, and T. Patarnello. 2002. Strategies for microsatellite isolation: A review. Mol. Ecol. 1:1-16.

Zha, H.G., R.I. Milne, and H. Sunday. 2008. Morphological and molecular evidence of natural hybridization between two distantly related Rhododendron species from the SinoHimalaya. Bot. J. Linn. Soc. 156:119-129.

Zhang, J.L., C.Q. Zhang, L.M. Gao, J.B. Yang, and H.T. Li. 2007. Natural hybridization origin of Rhododendron agastum (Ericaceae) in Yunnan, China: Inferred from morphological and molecular evidence. J. Plant Res. 120:457-463. 